\title{
Imperceptible Watermarking Technique using Arnold's Transform and Cross Chaos Map in DCT Domain
}

\author{
Chittaranjan Pradhan \\ School of Computer \\ Engineering, \\ KIIT University, \\ Bhubaneswar, India
}

\author{
Vilakshan Saxena \\ School of Computer \\ Engineering, \\ KIIT University, \\ Bhubaneswar, India
}

\author{
Ajay Kumar Bisoi \\ School of Computer \\ Engineering, \\ KIIT University, \\ Bhubaneswar, India
}

\begin{abstract}
Digital watermarking is the process of embedding secret information into a digital signal which may be used to verify its authenticity or the identity of its owners. In this paper, we have taken image as the medium of watermarking. Here, we have discussed an imperceptible watermarking technique by using double encryptions in DCT domain. For the encryption process, Arnold's transform and Cross chaos map are used. Like other algorithms, this one is also evaluated by Peak signal to noise ratio (PSNR). From the experimental results, it can be proved that this algorithm gives better PSNR value, which makes the algorithm to imperceptible.
\end{abstract}

\section{General Terms}

Security.

\section{Keywords}

Watermarking, Arnold's transformation, Cross chaotic map, Discrete cosine transform.

\section{INTRODUCTION}

Due to the rapid growth in data communication and networking industry, huge multimedia data are used now a day over the public network, called internet. There fore, information security is a critical issue in the current era. For protecting valuable data from illegal access, different encryption techniques are used. But, when privacy and duplicity come into picture, these encryptions techniques alone cannot solve the problem. Instead, watermarking technique is used to solve this problem. Due to the copyright protection and privacy preservation, this technique has got much attention in the past decades [2, 8]. For this reason, many algorithms have been proposed such as perceptible watermarking and imperceptible watermarking, for digital media. As an important type of watermarking technique, imperceptible watermark embedded into a cover image by sophisticated algorithms and is invisible to the naked eye [2]. A signal may carry several different watermarks at the same time.

In this paper, initially the watermark image is encrypted using both Arnold's transformation and Cross chaos sequence. Similarly, the cover image is converted from the spatial domain to frequency domain using DCT equation; where the encrypted watermark signal is embedded.

\section{RELATED WORK}

In 2007, M. Al Baloshi, M. E. Al-Maulla have developed a DCT based watermarking technique for image authentication. This technique embeds one watermark bit in each DCT block by shifting a randomly selected coefficient to have a mapped value, in a coefficient-binary-mapping function, which is identical to the watermark bit [6].In 2011, Jianhu Song and
Yong Zhu developed a watermarking technique based on double encryptions in DCT domain. For the encryption, they have taken Arnold's transform and 1D logistic chaos methods. After encrypting the watermark, the signal will be embedded into the transformed domain of the cover image [4].

\section{ARNOLD'S TRANSFORMATION}

According to Arnold's transformation, an image is hit with the transformation that apparently randomizes the original organization of its pixels. However, if iterated enough times, eventually the original image reappears. The number of iterations taken is known as the Arnold's period. The period depends on the image size; i.e. for different size images, Arnold's period will be different [3].

$\left(\begin{array}{l}x^{\prime} \\ y^{\prime}\end{array}\right)=\left(\begin{array}{ll}1 & 1 \\ 1 & 2\end{array}\right)\left(\begin{array}{l}x \\ y\end{array}\right) \bmod n$

where $n$ is the size of the image.

Equation 1 is used to transform each and every pixel coordinates of the image. When all the coordinates are transformed, the image we obtain is a scrambled image. At a certain step of iterations, if the image we achieve reaches our anticipated target (i.e. up to secret key), we have achieved the scrambled image we wanted to. The decryption of image relies on the transformation periods (i.e. the number of iteration to be followed $=$ Arnold's period - secret key). Let us apply Arnold's transformation to the image moon_64.bmp by taking Arnold's key as 20. Figure 1(a) shows the original image and Figure 1(b) shows the Arnold's encrypted image.

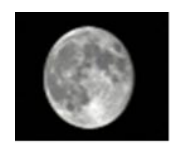

a

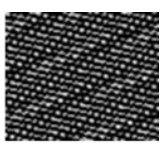

b
Figure 1. Arnold's transformation

\section{CROSS CHAOTIC MAP}

Chaotic map is used for randomization purpose. Cross Chaotic map is defined as:

$\left\{\begin{array}{l}x_{i+1}=1-\mu * y_{i}^{2} ; x, y \in[-1,1] \\ y_{i+1}=\cos \left(k \cos ^{-1} x_{i}\right)\end{array}\right.$

where variables $\mu$ and $k$ are the control parameters of the system. But, the system will show better chaotic behavior when $\mu=2$ and $\mathrm{k}=6$.

Two chaotic sequences $\mathrm{X}=\mathrm{x}_{0}, \mathrm{x}_{1} \ldots$ and $\mathrm{Y}=\mathrm{y}_{0}, \mathrm{y}_{1} \ldots$ using initial values $\mathrm{x}_{0}, \mathrm{y}_{0}$ and control parameters $\mu$ and $\mathrm{k}$, are generated. $\mathrm{X}$ and $\mathrm{Y}$ are reconstructed as row and column matrix respectively. Then they are multiplied with each other, to get a new matrix k'. Finally this matrix converted to binary 
matrix using equation 3. Due to increased number of parameters, this technique gives higher security as compared to the 1D logistic map [5]. This motivates us to work with Cross Chaos map instead of the standard 1D logistic map sequence.

$F(x)=\left\{\begin{array}{l}0,0<z(i, j) \leq 0.5 \\ 1,0.5<z(i, j) \leq 1\end{array}\right.$

Let us apply Cross chaos transformation to the image moon_64.bmp by taking control parameters as $\mu=2$ and $\mathrm{k}=6$. Figure 2(a) shows the original image and Figure 2(b) shows the Cross chaos encrypted image.

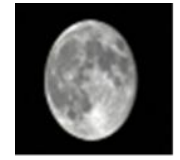

a

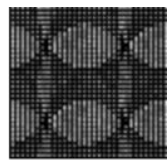

b
Figure 2. Cross chaos transformation

\section{DISCRETE COSINE TRANSFORM}

Discrete Cosine Transform (DCT) is a method to convert a signal from the spatial domain to frequency domain or transform domain. Here, DCT transforms the cover image of size $\mathrm{M} \times \mathrm{N}$ pixels to frequency representation, by grouping the pixels into non-overlapping blocks of $8 \times 8$ pixels and transforming the pixels blocks into 64 DCT coefficients each. In particular, DCT is a Fourier related transform similar to the Discrete Fourier Transform (DFT), but using only real numbers $[1,7]$.

Let $\mathrm{F}(\mathrm{x}, \mathrm{y})$ denote an 8-bit grayscale image with $\mathrm{x}=1,2 \ldots \mathrm{M}_{1}$ and $\mathrm{y}=1,2 \ldots \mathrm{N}_{1}$. This image is divided into $8 \times 8$ blocks and two dimensional (2D) DCT is performed on each of $\mathrm{L}=\mathrm{M}_{1} \mathrm{x}$ $\mathrm{N}_{1} / 64$ blocks. The mathematical definition of 2D DCT is:

$$
F(u, v)=\frac{\frac{1}{4} C(u) C(v) \sum_{x=0}^{7} \sum_{y=0}^{7} F(x, y) \cos \left[\frac{\pi(2 * x+1) u}{16}\right]}{\cos \left[\frac{\pi(2 * y+1) v}{16}\right]}
$$

where $\mathrm{u}=0 \ldots 7, \mathrm{v}=0 \ldots 7$ and

$$
C(k)=\left\{\begin{array}{l}
\frac{1}{\sqrt{2}} \text { for } k=0 \\
1, \text { otherwise }
\end{array}\right.
$$

Let us apply 2D DCT to the image Baboon.bmp. Figure 3(a) shows the original image and Figure 3(b) shows the DCT transformed image.

To convert the signal from frequency domain again into spatial domain, the Inverse DCT will be used.

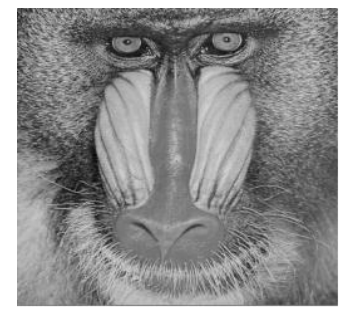

a

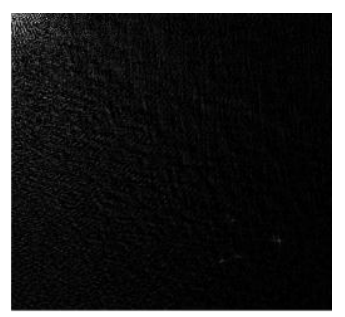

b
Figure 3. DCT transformation
The expression for Inverse DCT is:

$$
F(x, y)=\begin{aligned}
& \frac{1}{4} \sum_{u=0}^{7} \sum_{v=0}^{7} C(u) C(v) F(u, v) \cos \left[\frac{\pi(2 * x+1) u}{16}\right] \\
& \cos \left[\frac{\pi(2 * y+1) v}{16}\right]
\end{aligned}
$$

\section{PROPOSED ALGORITHM}

In this paper, we propose an algorithm for imperceptible watermarking using Arnold's Transformation, Cross Chaotic Map and Discrete Cosine Transform (DCT) techniques.

\subsection{Proposed Embedding Algorithm}

Let $\mathrm{X}$ is the original image of size $\mathrm{M} \times \mathrm{M}$ and $\mathrm{w}$ be the watermark image of size $\mathrm{N} \times \mathrm{N}$ then the watermark embedding algorithm is as follows:

1. Encrypt the watermark image w with Arnold's Transformation with the help of equation 1 up to the Arnold's key which is calculated on the basis of size of the watermark.

2. Use the encrypted watermark as the input to the Cross Chaotic Map and encrypt it using equation 2 to generate the final encrypted watermark.

3. Take the original cover image $X$ and divide it into non-overlapping blocks of size $8 \times 8$ and apply 2D DCT on each of the blocks of the cover image to convert it from spatial to transform domain using equation 4.

4. Embed the watermark generated in step 2 pixel by pixel into each of the $8 \times 8$ DCT blocks as per equation 7.

$$
F(x, y)=\alpha * w(i)
$$

where $\mathrm{F}(\mathrm{x}, \mathrm{y})$ represents each of the $8 \mathrm{x} 8$ blocks, $\mathrm{w}(\mathrm{i})$ represents the target watermark taken in row major order and $\alpha$ stands for the watermarking strength. Lower the value of $\alpha$, better the quality of the watermark image.

5. After embedding the watermark, reconstruct the original cover image using the process of Inverse DCT using equation 6.

\subsection{Proposed Extraction Algorithm}

The extracting algorithm is the opposite process of embedding to recover the original watermark embedded in the embedding process. The process is as follows:

1. Take the watermarked cover image as input and apply DCT to it to convert it into transform domain. Then, extract the embedded watermark pixel by pixel from each $8 \times 8$ block using equation 8 .

$w(i)=\frac{F(x, y)}{\propto}$

where w(i) represents extracted watermark, $F(x, y)$ represents each $8 \times 8$ DCT block and $\alpha$ represents strength of watermarking.

2. Use the extracted watermark as input for the process of Cross Chaotic decryption and decrypt the watermark using equation 2 .

3. Apply Arnold's Transformation to the result of step 2 to decrypt the resultant image according to calculated period using equation 1 .

4. After Arnold's Transformation, the resultant watermark image is our desired image. 


\section{EXPERIMENTAL RESULTS}

The performance of the watermarked image can be evaluated on the basis of the peak signal to noise ratio (PSNR) in decibels $(\mathrm{dB})$ as given in equation 10. Higher the values of PSNR, better is the quality of the watermarked image. PSNR more than $30 \mathrm{dBs}$ is considered to be an acceptable quality image, in which watermark causes no major alteration to the quality of the image [2].

$$
\begin{aligned}
& M S E=\frac{1}{m n} \sum_{i=0}^{m-1} \sum_{j=0}^{n-1}[I(i, j)-K(i, j)]^{2} \\
& P S N R=10 \log _{10}\left(\frac{R}{M S E}\right)
\end{aligned}
$$

where MSE is the Mean Square Error of the watermarked image and the original image and $\mathrm{m}, \mathrm{n}$ are the number of rows and columns. I and $\mathrm{K}$ are the original and watermarked image respectively.

For the experimental analysis, we have taken moon_64.bmp as the watermark signal as shown in Figure 4(a). This image is of size 64 x 64, whose Arnold's period is 96. In the current experiment, we have taken 40 as the Arnold's key. The Arnold's transformed signal is shown in Figure 4(b). Similarly, by taking $\mu=2$ and $\mathrm{k}=6$, we got the Cross Chaos encrypted signal as shown in Figure 4(c). Here, Baboon.bmp is taken as the cover image, which is of size $512 \times 512$ (shown in Figure $4(d))$. Here, the number of $8 \times 8$ blocks is $(512 \times 512) /$ $(8 \times 8)=4096$. If we'll hide 1 pixel of the message into 1 block, then the maximum number of pixels that can be hidden is 4096 . As our message is of $64 \times 64$, thus we are able to hide the whole message (encrypted format) into the cover image. When 2D DCT is applied on the cover image, the transformed image is similar to the image shown in Figure 4(e). By applying the equation 7 for data embedding as well as the Inverse DCT, the watermarked cover image is shown in Figure 4(f).
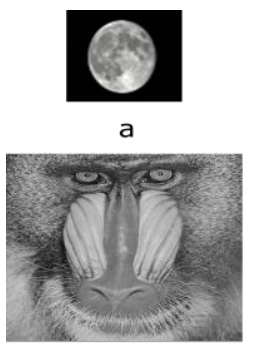

d
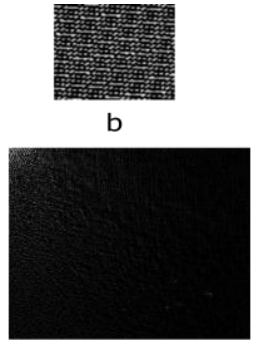

e
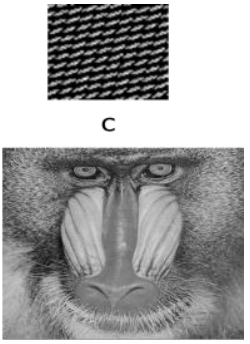

f
Figure 4. Watermark embedding process (moon_64)

When we'll compare both the original and watermarked cover images shown in Figure 4(d) and 4(f) respectively, the resultant PSNR value is $+43.82 \mathrm{~dB}$.

When the watermarked cover image (shown in Figure 5(a)) is applied with 2D DCT, the transformed image is found as shown in Figure 5(b). When we'll extract all the pixels from the transformed domain, the extracted watermark will be same as the figure shown in Figure 5(c). After the application of Cross Chaos decryption process, the extracted watermark will be as shown in Figure 5(d). When the Arnold's transformation is done on this extracted signal for the remaining number of iterations (i.e. Arnold's period - Arnold's key), the resultant image is similar to the original watermark image as shown in Figure 5(e).
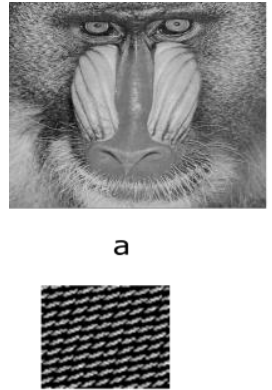

C
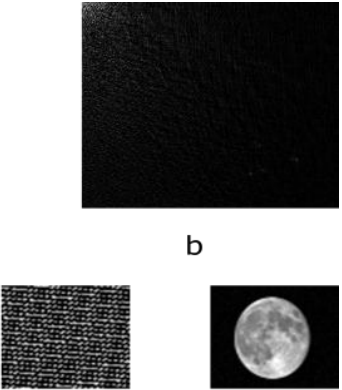

d

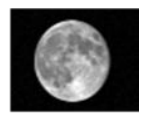

Figure 5. Watermark extraction process (moon_64)

Similarly, we have tested our algorithm by taking another message called, lena.bmp. This image has Arnold's period of 96, out of which we have taken 60 as the Arnold's key. Similarly, the control parameters for Cross chaos sequence are taken as $\mu=2$ and $\mathrm{k}=6$. By taking the same Baboon.bmp as the cover image, we have applied the embedding process to the message. The different stages of the embedding are displayed in Figure 6. This different parts of this figure shows the original message, Arnold's encrypted message, Arnold'sCross chaos encrypted message, original cover, DCT version of cover and the watermarked cover respectively. Here, we have got the PSNR value as $+41.53 \mathrm{~dB}$.

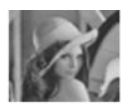

a

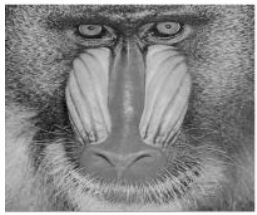

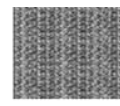

b

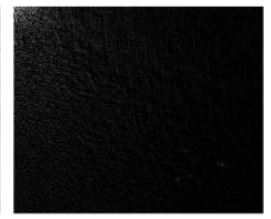

e

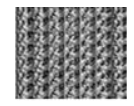

C
Figure 6. Watermark embedding (lena)

We have also tested the watermark extraction process to the watermarked cover, where lena.bmp image has been hidden Figure 7 shows this extraction process. Figure 7(a) $-7(\mathrm{e})$ shows the watermarked cover, DCT version of cover, extracted encrypted message, extracted Cross chaos decrypted message and the extracted Arnold's decrypted message respectively.

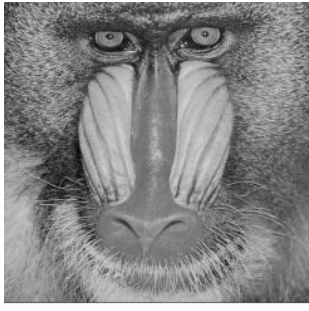

a

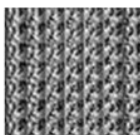

C

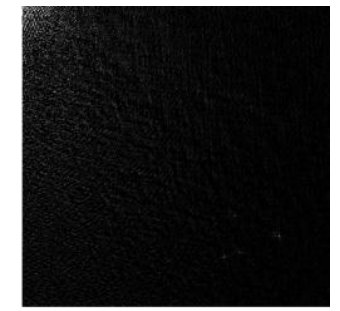

b

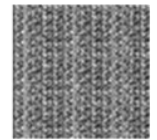

d

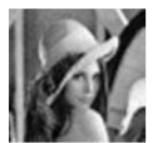

e
Figure 7. Watermark extraction (lena) 
When we'll compare our algorithm with the algorithm described by Jianhu Song and Yong Zhu, we find the PSNR value produced by our algorithm is larger to the fomer as shown in Table 1. Also, due to the better randomness of cross chaos sequence as compared to $1 \mathrm{D}$ logistic chaos sequence [5], our technique provides better randomness at the time of encryption.

\section{Table 1. PSNR values}

\begin{tabular}{|l|l|l|}
\hline \multicolumn{1}{|c|}{ Image } & \multicolumn{1}{|c|}{$\begin{array}{c}\text { PSNR of Proposed } \\
\text { algorithm }\end{array}$} & $\begin{array}{c}\text { PSNR of Referenced } \\
\text { algorithm }\end{array}$ \\
\hline moon_64 & $+43.82 \mathrm{~dB}$ & $+41.73 \mathrm{~dB}$ \\
\hline lena & $+41.53 \mathrm{~dB}$ & $+40.33 \mathrm{~dB}$ \\
\hline
\end{tabular}

\section{CONCLUSIONS}

In this proposed algorithm, we have used a combined approach of Arnold's Transformation and Cross Chaotic Map to encrypt the watermark. As it depicts double level encryption technique, so it is more secure as compared to the single level encryption techniques. Similarly, due to more randomness in case of Cross chaos map, it is more secured than 1D logistic chaotic map. As far as imperceptibility nature of the proposed algorithm is concerned, we derived the PSNR value of two different watermarks and compared them which turned out to be more than 40 that mean it is difficult to distinguish between original and watermarked image. Thus, we can conclude that the proposed algorithm is an effective and secure algorithm. In future, this algorithm can be tested with various attacks for the robustness.

\section{REFERENCES}

[1] A. Nag, S. Biswas, D. Sarkar, P. P. Sarkar, "A novel technique for image steganography based on Block-DCT and Huffman Encoding", International Journal of Computer Science and Information Technology, June 2010, vol. 2, no. 3, pp.103-112

[2] Frank Y. Shih, Digital Watermarking and Steganography, CRC Press, 2012, isbn: 1-4200-4757-4

[3] Gabriel Peterson, "Arnold's cat map survey", Math 45Linear Algebra, Fall 1997, pp.1-7

[4] Jianhu Song and Yong Zhu, "A Digital Watermarking Method by Double Encryption Based on Arnold and Chaos in DCT domain", Applied Mechanics and Materials, 2011, vol. 65, pp.104-107

[5] Kuldeep Singh, Komalpreet Kaur, "Image Encryption using Chaotic Maps and DNA Addition Operation and Noise Effects on it", International Journal of Computer Applications, June 2011, vol. 23, no.6, pp. 17-24

[6] M. Al Baloshi, M. E. Al-Maulla, "A DCT-Based Watermarking Technique for Image Authentication", International Conference on Computer Systems and Applications", IEEE, 2007, pp. 754-760

[7] S. Singh, T. J. Siddiqui, R. Singh, H. V. Singh, "DCTdomain robust data hiding using chaotic sequence", International Conference on Multimedia, Signal Processing and Communication Technologies, IEEE, 2011, pp. 300-303

[8] Siddharth Singh, Tanveer J. Siddiqui, “A Security Enhanced Robust Steganography Algorithm for Data Hiding", International Journal of Computer Science Issues, May 2012, vol. 9, issue. 3, no. 1, pp. 131-139 